\title{
Molecular docking studies of phytochemicals against trehalose-6- phosphate phosphatases of pathogenic microbes
}

\author{
H. R. Umesh ${ }^{1 *}$ (D, K. V. Ramesh ${ }^{2}$ and K. S. Devaraju ${ }^{3}$
}

\begin{abstract}
Background: Many of the pathogenic microbes use trehalose-6-phosphate phosphatase (TPP) enzymes for biosynthesis of sugar trehalose from trehalose-6-phosphate (T6P) in their pathway of infection and proliferation. Therefore, the present work is an approach to design new generation candidate drugs to inhibit TPP through in silico methods.

Results: Blast P and Clustal Omega phylogenetic analysis of TPP sequences were done for 12 organisms that indicate and confirm the presence of three conserved active site regions of known TPPs. Docking studies of 3D model of TPP with 17 phytochemicals revealed most of them have good binding affinity to an enzyme with rutin exhibiting highest affinity (Binding energy of $-7 \mathrm{kcal} / \mathrm{mole}$ ). It has been found that during docking, phytochemical leads bind to active site region 3 of TPP sequences which coordinates Mg2+ and essential for catalysis.

Conclusions: Binding poses and distance measurement of TPP-phytochemical complexes of rutin, carpaine, stigmasterol, $\beta$-caryophyllene, and a-eudesmol reveals that the lead phytochemicals were in close proximity with most of the active site amino acids of region 3 (distance range from 1.796 to $2.747 \mathrm{~A}^{\circ}$ ). This confirms the tight binding between enzyme and leads which may pave way for the discovery of new generation drugs against TPP producing pathogenic microbes to manage diseases.
\end{abstract}

Keywords: Trehalose-6-phosphate phosphatase, Binding affinity, In silico, Phytochemical, Rutin

\section{Background}

Stress protectant molecule trehalose is an essential sugar in most organisms other than mammals [1]. The critical pathway of trehalose biosynthesis involves consecutive reactions of trehalose phosphate synthase (TPS) and trehalose-6-phosphate phosphatase (TPP) [2]. Humans and animals are affected by virulent nature of the above pathway. Especially that of TPPs which forms sugar from trehalose-6-phosphate (T6P) exhibit virulent functions in a number of ways in different microbes. Many serious diseases such as tuberculosis, Aspergillosis, Candedemias, and nematode diseases are main cause of mortality

\footnotetext{
* Correspondence: umeshhr_76@ymail.com

${ }^{1}$ Research and Development Centre, Bharathiar University, Coimbatore 641046, India

Full list of author information is available at the end of the article
}

worldwide each year, mostly mediated by TPPs of concerned pathogens [3-10].

Trehalose derived from TPP of Mycobacterium is required for the synthesis of trehalose mono mycolate and trehalose di mycolate responsible for infection and survival of pathogen in affected animals [11-13]. Trehalose mycolates of variable chain length in few pathogens are required for the evasion of the host immune system. Candedemias causing fungi need TPP for various phases of life cycle and virulent functions [14-16]. In order to respond to stress-related issues and infection, Aspergillus organisms depend on TPPs. Elephantiasis causing nematodes requires Trehalose for various cell survival and transmission purposes [9]. In Cryptococcus neoformans, trehalose released by TPP is involved in stress protection and host survival mechanisms [3]. Overall, one may conclude that trehalose 
phosphate phosphatases are implicated as main virulent molecules in most of the above pathogenic organisms.

Antibiotic resistance or drug resistance developed by pathogens is a common problem of modern medicine related to abuse of drugs. To find the solution to the above problem, targeting the pathways or genes common to pathogens but totally absent in hosts holds the key. Though specific drugs are available, trehalose pathway and the genes like that of TPP can be tried as better anti-pathogenic targets which specifically affect pathogens but not humans and animal hosts. To achieve this objective, characterization of TPP enzymes and specific inhibitor design against them is fastly emerging as a very active area of biomedical research. In this regard, we have previously purified and characterized a TPP from maize genotype EC 558706 [46]. Therapeutic potential of TPP inhibitors in treating tuberculosis and other bacterial, fungal, and parasitic diseases have not yet been fully dealt.

The non-virulent TPPs of plants and virulent TPPs of microbes originally belong to Halo acid dehalogenasesrelated superfamily of proteins which binds T6P specifically. The highly conserved catalytic motifs are regular features of TPPs of HAD superfamily. The potential drug candidates designed against these TPPs may act by making links with highly conserved catalytic amino acids and thereby obstructing the development and virulent-related functions of pathogens but the host is unaffected [17].

Plant-based phytochemicals offer attractive, effective, and holistic drug action against the pathogens without much of the side effects. Hence, we are interested in screening the potential phytochemicals as drug molecules against TPPs and to look into their effectiveness against pathogenic microbes. Structure-based drug designing using phytochemicals can be used to reduce the ambiguity involved and fasten up the process [18]. Hence, the present study focuses on in silico docking work on selected phytochemicals as lead molecules to check their binding affinities to modeled TPP protein in comparison to known antimicrobial drugs. This may help to come out with leads of next-generation drugs against virulent TPPs involved in infection and proliferation of many pathogens.

\section{Methods}

\subsection{Selection, modeling, and drug-likeness test of phytochemicals for drug design}

\subsubsection{Selection of phytochemicals from medicinal plants for} inhibitor design

The 5 of 21 medicinal plants were selected for the identification of phytochemicals based on their inhibitory property against previously purified maize TPP by us. The following are the medicinal plants with parts for the studies: (1) Aegle marmelos (leaves); (2) Ocimum basilicum (leaf); (3) Ribes nigrum (fruit); (4) Carica papaya (fruit); and (5) Costus pictus (leaves). More than 17 phytochemicals from above said plants (possessing antibacterial, antifungal, nematicidal, antiviral, antioxidant, antituberculosis, cancer-preventive effects) were retrieved from extensive literature survey for ligand (inhibitor) preparation to act against TPPs of pathogens [19-24]. Their respective two-dimensional chemical structures in structured data format (SDF) were retrieved from PubChem-NCBI database and SDF format was converted into Protein data bank (PDB) format using Pymol for further analysis. The chemical structure of natural substrate trehalose-6-phosphate and antibacterial such as ampicillin and antifungal drugs such as isoniazid and fluconazole were processed similarly as controls.

\subsubsection{Drug-likeness test of phytochemicals based on the Lipinski rule using Schrodinger software}

To perform docking studies, the 17 phytochemicals retrieved from five medicinal plants should satisfy druglikeness test (determines whether particular compound could be used as the drug). The evaluation of druglikeness test for all compounds was carried out using qikprop tool of Schrodinger drug discovery software [25]. Qikprop is a quick, accurate, easy to use absorption, distribution, metabolic, and excretion prediction program of Schrodinger software. It predicts pharmaceutically relevant properties of organic molecules individually or in batches. This tool evaluates the compounds based on the Lipinski rule, which states that an active oral drug should qualify the following criteria: molecular weight should be in the range from 130 to $725 \mathrm{Da}, \log \mathrm{P}$ should be $<5$, H-bond donor should be 0 6 , H-bond acceptor should be 2-20, number of rotatable bonds should be $>5$. Along with drug-likeness test, molecular volume for the compounds should be between 500 and 2000 [26].

\subsubsection{Development of phytochemical models for docking}

The predicted Lipinski values (data sets) of 17 compounds for TPP were used for the development of the phytochemical models. The qikprop result analysis studies were carried out to correlate datasets-descriptors of 17 plant compounds along with 3 prescribed drugs for specific TPP inhibitory activity. The descriptors of compound ( $\log \mathrm{P}$, molecular weight, $\mathrm{H}$-bond donor, $\mathrm{H}$-bond acceptor, number of rotatable bonds, molecular volume) were compared manually with the qikprop standard values in addition to volume. The phytochemicals which exhibit maximums number of properties within the range of standard values were selected for further docking studies with 3D structure of representative TPP enzyme. 
2.2 Conserved motif detection, phylogenetic tree construction, and in silico homology modeling of TPP 2.2.1 Identification of conserved active site regions for TPP group of proteins

TPP sequences from various plants (Oryza. sativa, Arabidopsis. thaliana, Zea.mays), pathogenic bacteria (Mycobacterium tuberculosis, Corynebacterium glutamicum), fungi (Sacharomyces cerevisiae, Candida albicans, Aspergillus niger, Aspergillus nidulans, Cryptococcus neoformans), and nematodes ( Caenorhabditis elegans, Brugia malayi) were retrieved by running protein BLAST (tool of NCBI) analysis based on IU02 TPP Sequence (Thermoplasma acidophilum) from PDB (protein data bank) submitted in NCBI. Further multiple sequence alignment (MSA) of above sequences using Clustal Omega program of EMBL [27] gives us the information about conservation of the binding site and active site amino acids of TPP group of proteins.

\subsubsection{Phylogenetic tree construction of TPPs from different organisms:}

ClustalW2 is an online tool of the European Bioinformatics Institute to determine the phylogenetic tree of protein sequences from a variety of organisms. Multiple sequence alignment of TPP protein sequences of various organisms were submitted to Clustal W2 package in fasta format. The following parameters were set. Tree format was Clustal and distance correction was on. Gaps in the sequences were excluded and clustering was done using neighbor-joining method. The output phylogenetic tree showing the specific evolutionary relationships was obtained from the EBI server for analysis [28].

\subsubsection{Homology model construction}

Though the 3D structure of TPP (1U02 of PDB) from $T$. acidophilum $[29,30]$ is readily available, we wanted to have a representative homology model for TPPs. As we have earlier purified a maize TPP, the amino acid sequence of a similar enzyme; Ramosa 3 (RA3) sequence was taken further to get a representative 3D model of all aligned TPPs from plant and pathogenic organisms [31]. RA 3 amino acid sequence was submitted to the Swiss modeler platform which has an identity at active sites with MSA sequences of TPPs of other organisms. Besides, we wanted to check whether the model protein has structural homology and can represent similar TPPs from plants, pathogenic bacteria, fungi, and nematodes. In the homology modeling project [32], Swiss model template library was searched with BLAST and HHBlits for evolutionarily related structures matching the target sequence. Homology model representing pathogenic and non-

Table 1 Dependent variables (descriptors) of phytochemicals estimated using Schrödinger's Qik-prop tool

\begin{tabular}{|c|c|c|c|c|c|c|c|c|}
\hline S. no & Compound name & PubChem ID & $\log P$ & Mol.Wt & $\mathrm{nOH}$ & $\mathrm{nOHNH}$ & $\mathrm{Nb}$ & Vol \\
\hline 1 & Beta caryophyllene & 5281515 & 5 & 208 & 0 & 0 & 0 & 788 \\
\hline 2 & Beta sitosterol & 222284 & 9.3 & 416 & 2 & 2 & 6 & 1439 \\
\hline 3 & Carpaine & 442630 & 6.3 & 482 & 10 & 4 & 0 & 1525 \\
\hline 4 & Catalposide & 93039 & -1 & 492 & 19 & 6 & 7 & 1286 \\
\hline 5 & Chavicol & 68148 & 4 & 156 & 2 & 0 & 3 & 563 \\
\hline 6 & 4-methyl hydroxy cinnamic acid & 445858 & 2 & 188 & 5 & 2 & 3 & 610 \\
\hline 7 & a-eudesmol & 92762 & 5 & 224 & 2 & 2 & 2 & 830 \\
\hline 8 & a-lonone & 5282108 & 3 & 198 & 1 & 0 & 2 & 765 \\
\hline 9 & Kaempferol & 5280863 & 2 & 302 & 10 & 5 & 1 & 794 \\
\hline 10 & Myricitin & 5281672 & 5 & 334 & 14 & 7 & 1 & 826 \\
\hline 11 & Myristic acid & 11005 & 5.3 & 230 & 4 & 2 & 12 & 1020 \\
\hline 12 & Quercetin & 5280343 & 2 & 318 & 12 & 6 & 2 & 797 \\
\hline 13 & Rutin & 5280805 & 1 & 626 & 20 & 11 & 6 & 1521 \\
\hline 14 & Stigmasterol & 5280794 & 8.6 & 416 & 2 & 2 & 5 & 1440 \\
\hline 15 & a-terpinol & 17100 & 2 & 156 & 1 & 1 & 1 & 632 \\
\hline 16 & Terpinoline & 11463 & 3 & 140 & 0 & 0 & 0 & 601 \\
\hline 17 & 3,5 diformyl 2,4,6 Trihydroxy toluene & 854656 & 4 & 196 & 2 & 0 & 6 & 212 \\
\hline 18 & Ampicillin & 6249 & -1 & 361 & 10 & 7 & 4 & 1040 \\
\hline 19 & Fluconazole & 3365 & 0.4 & 320 & 7 & 1 & 5 & 935 \\
\hline 20 & Isoniazid & 3767 & -0.7 & 145 & 3 & 2 & 1 & 487 \\
\hline
\end{tabular}

Note: Log P lipophilicity, Mol.Wt molecular weight, $n \mathrm{OH}$ no. of $\mathrm{H}$ bond acceptors, $n \mathrm{OHNH}$ no. of $\mathrm{H}$ bond donors, $n b$ no. of rotatabe bonds 
pathogenic organisms was obtained and compared with $1 \mathrm{U} 02$.

\subsection{Docking studies using AutoDock Vina 4.2 2.3.1 Preparation of modeled TPP and 17 ligands for docking}

The representative homology model of TPP molecule from Swiss model server did not have a complete charge assigned to them. Hence, before docking, polar hydrogens were added to the macromolecules and then assigned the partial atomic charges using AutoDock Vina 4.2 [33]. The non-polar and polar hydrogen atoms were merged. For 17 ligands along with conventional drugs for comparison, Gasteiger charges were added, non-polar hydrogens were merged and also rotatable bonds were determined based on the nature of ligand molecule. TORSDOF was used to calculate the change in free energy $(\delta G)$ caused by the loss of torsional degree of freedom upon binding. The atomic fragmental volume and the atomic solvation parameters were used here to calculate the energy contribution of the desolvation of the macromolecules by ligand binding. Peptide backbone bonds were constructed. Bonds between selected atoms and all the active bonds are made rotatable [34]. Grid maps were generated and spacing was adjusted to $0.8 \AA$ to enable ligand binding. The grid dimension was adjusted to $40 \times 40 \times 40$ points. AutoDock Vina uses interaction maps for docking. Prior to the actual docking run, these maps were calculated by Auto Grid. For each ligand atom type, the interaction energy between the ligand atom and receptor was calculated for the entire binding site, which is discretized through a grid. The protein was embedded in a $3 \mathrm{D}$ grid and a probe was placed at each grid point. The interaction energy of the protein was assigned at each grid point and the affinity for each of the ligand was calculated.

\subsubsection{Docking protein and ligand molecules}

Automated docking software AutoDock Vina [33] 4.2 was used to evaluate binding affinity of ligands (with drug-likeness property) and three prescribed drugs (ampicillin, fluconazole, and isoniazid) to homology model of TPP enzyme. Docking energy of all ligand molecules and drugs, substrate-T6P (trehalose-6-phosphate) were evaluated by using empirical-free energy functions and Lamarckian genetic algorithm. These tools calculate binding-free energy $(\delta G)$ based on different electrostatic, Vander Waal, hydrogen bonding, and desolvation effects. Docking precision was set to "regular precision" and "flexible" ligand-docking mode was employed for each docking run. The stability of each

Table 2 Drug-likeness score of different phytochemicals based on Lipinski rule

\begin{tabular}{|c|c|c|c|c|c|}
\hline Sl. no. in Table 1 & Sl. no. & Compound name & Pubchem ID & No. of violations & Drug-likeness score \\
\hline 13 & 1 & Rutin & 5280805 & 1 & 38 \\
\hline 4 & 2 & Catalposide & 93039 & 1 & 33 \\
\hline 10 & 3 & Myricitin & 5281672 & 1 & 29 \\
\hline 11 & 4 & Myristic acid & 11005 & 0 & 24 \\
\hline 12 & 5 & Quercetin & 5280343 & 1 & 23 \\
\hline 3 & 6 & Carpaine & 442630 & 1 & 21 \\
\hline 14 & 7 & Stigmasterol & 5280794 & 0 & 20 \\
\hline 2 & 8 & Beta-sitosterol & 222284 & 0 & 20 \\
\hline 9 & 9 & Kaempferol & 5280863 & 2 & 19 \\
\hline 17 & 10 & 3,5 diformyl 2,4,6 Trihydroxy toluene & 854656 & 2 & 14 \\
\hline 6 & 11 & Cinnamic acid & 445858 & 1 & 13 \\
\hline 7 & 12 & a-eudesmol & 92762 & 1 & 12 \\
\hline 5 & 13 & Chavicol & 68148 & 1 & 11 \\
\hline 8 & 14 & a-lonone & 5282108 & 2 & 7 \\
\hline 1 & 15 & $\beta$-caryophyllene & 5281515 & 2 & 6 \\
\hline 15 & 16 & $\mathbf{a}$-terpineol & 17100 & 2 & 6 \\
\hline 16 & 17 & Terpinoline & 11463 & 3 & 4 \\
\hline 18 & 18 & Ampicillin & 6249 & 1 & 22 \\
\hline 19 & 19 & Fluconazole & 3365 & 1 & 14 \\
\hline 20 & 20 & Isoniazid & 3767 & 2 & 8 \\
\hline
\end{tabular}


A

B.malayi
C.elegans1 I
C. Gob
C.neoformans
A.nidulang
A.niger
C.albican
S. cerevigiae
C.glutamicum
M.tuberculosis
Z.mays
A.thaliana
O. sativa

yhpk--gketflk-eyedtvkflktfisseai tgkkpifit lwdgt nkdycsqyatnlqp fv-d--deirfek-evaeckaflmdlidtsttggikplfit twdgthkdycsqyatnl fv-d--deirfek-evaeckaflmdlidtsttggikplfit dwdgt pkdycsqyatnlqp ------vgg--ehtahhtpaldtaqf taykkankrlil dydgt tpivkvoshav------19gfd--qsmatpaldrtkllkqyrks krl fmf dydgt tplvkdpqsai--

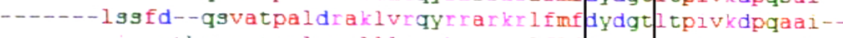
------91--vthsnзytpalnrpli innykesqrifl fydgt tpivqdpaai--

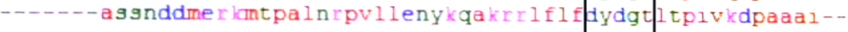

ge-vgurtgd lse--vgvrtgdrrmgqlpdalqalg-ladglvarqpavf fafdg tl sdlvedpdaaw------adaehgdwme - khpsa laqfepl laaa kg kqi vinf 1 dydg 1 spivedpdrav-lsndatdintyrewmq l kypsa l tofe kimg fa kg krial fl dydgt l spiveepdcay-nsseeedpaysawma-kcpsalasfkqivasaqgkkiavfl dydgt lspivddpdkav--

B

B.malayi

C.elegans1

C.Gob

C.neoformans

A.nidulans

A.niger

C.albican

S.cerevisiae

C.glutamicum

M. tuberculosis

2. mays

A. thaliana

O.gativa

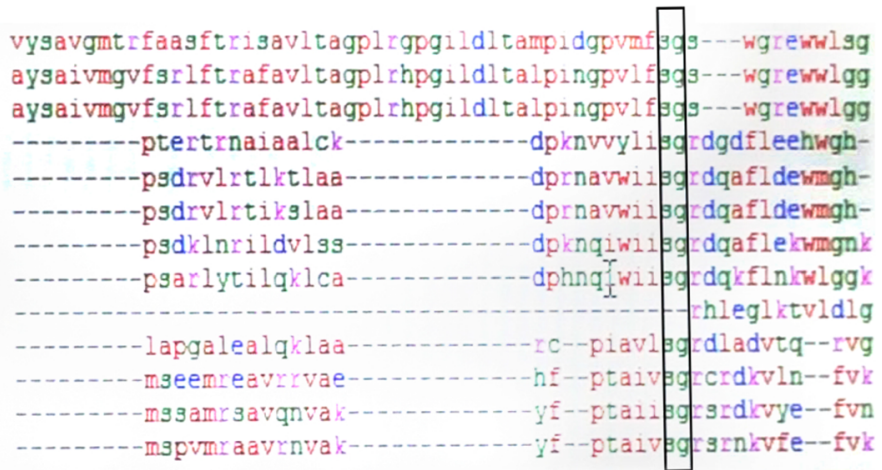

C

B.malayi
C.eleganst
C. Gob
C.neoformans
A.nidulans
A.niger
C. albican
S. cerevisiae
C.glutamicum
M. tuberculosis
2.mays
A.thaliana
O.sativa

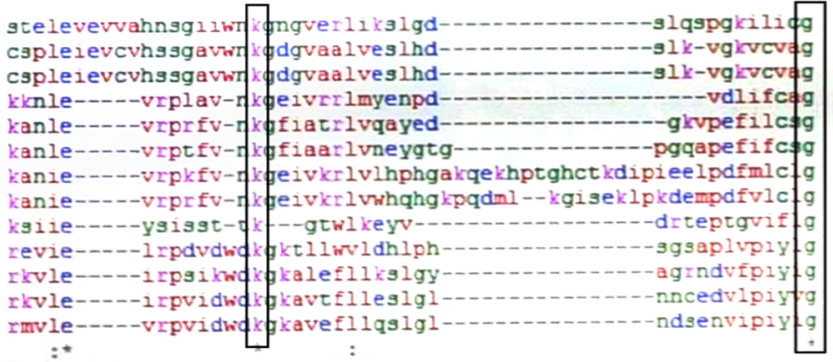

B.malayi
C.elegangl
C. Gob.
C.neoformans
A.nidulans
A.niger
C.albican
S. cerevisiae
C.glutamicum
M.tuberculosis
Z.mays
A. thaliana
O.sativa

\begin{tabular}{|c|c|}
\hline $\begin{array}{l}d t l s d \\
d t a s d \\
d t a s d \\
d d k t d \\
d d f \tau d \\
d d f t d \\
d d l t d \\
d d f t d \\
d d t \tau d \\
d d i t d \\
d d\end{array}$ & 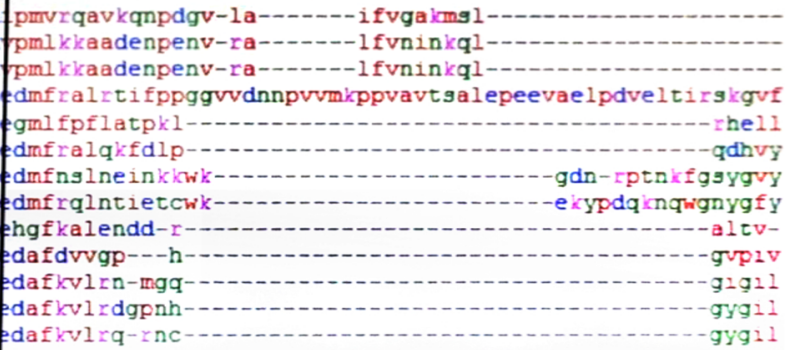 \\
\hline
\end{tabular}

Fig. 1 a Region 1 of conserved amino acid sequences of TPP proteins. $\mathbf{b}$ Region 2 of conserved amino acid sequences of TPP proteins. $\mathbf{c}$ Region 3 of conserved amino acid sequences of TPP proteins 


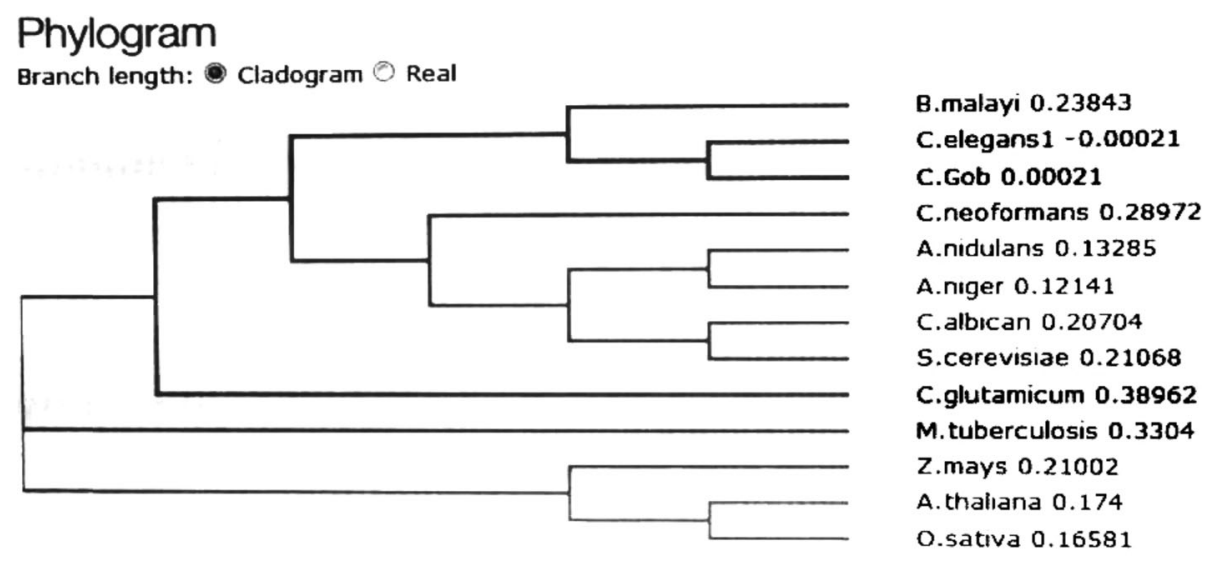

FMAI Firmnaan

Fig. 2 Phylogram of MSA of various TPP proteins

docked pose was evaluated using Auto dock energy calculations [35].

\subsubsection{Model TPP docking poses with lead phytochemicals, prescribed drugs, and substrate trehalose-6-phosphate} Blast P and MSA of various TPP sequences assisted us to confirm the conservation of active site regions. It is known that HAD family of magnesium-dependent phosphatases like TPPs have a common reaction mechanism due to conserved active site regions. Further, we wanted to know the proximity of binding of each of the ligand on conserved regions in model TPP. In this regard, we have taken docking poses of TPP binding by 17 ligands. We have represented five of them (at least one representative from five plant extracts with highest binding energy), three prescribed drugs and substrate trehalose phosphate.

\section{Results}

\subsection{In silico studies}

3.1.1 Selection and development of phytochemical models of ligands for inhibitor design

Usually, phytochemicals are known to have one or more medicinal properties. For proper docking during the in silico drug designing protocol, there is a need for phytochemicals to qualify the drug-likeness test, i.e., they have to be in the range of Lipinski rule for various descriptors. The independent descriptors were determined for each of the 17 phytochemicals using Schrodinger software's Qikprop program and represented in Table 1. A minimum of two violations are allowed to calculate the drug-likeness score. The different descriptors for each phytochemical (ligand) along with 3 prescribed drugs were manually compared with standard values and finally drug-likeness scores were computed as shown in Table 1. According to Table 2, Rutin has the highest likeness score of 38 whereas terpinolene has the least score of 6 . Ligands were segregated into zero violators (myristic acid, stigmasterol, beta-sitosterol). In addition to rutin and carpaine, many compounds were one violators and the rest of them are two violators. As the standard drug isoniazid also violates two rules with drug-likeness score of 8 , we have decided to carry all the two rule violator phytochemicals for further analysis, i.e., for docking [36].

\subsection{Conserved motif detection, phylogenetic tree} construction, and in silico homology modeling of TPP 3.2.1 Identification of conserved active site regions for TPP group of proteins

Three highly conserved active site motifs of TPPs are as follows: region 1 is near to $\mathrm{N}$ terminal end, contains amino acid sequence DXDX (T/V) where the first aspartic residue forms a phosphorylated intermediate with the substrate while the second residue plays an important role in catalysis as shown in Fig. 1a. Region 2 has a conserved serine or threonine S/T (GX), which serves to form a hydrogen bond with the phosphate group of the substrate. Region 3 contains the $K(X) 16$ $45(\mathrm{G} / \mathrm{S})(\mathrm{D} / \mathrm{S}) \mathrm{D} / \mathrm{TXX}(\mathrm{D} / \mathrm{N})$ sequence being part of the

Table 3 Template TPP from PDB with specifications

\begin{tabular}{|c|c|c|c|c|c|c|c|c|}
\hline Template & $\begin{array}{l}\text { Seq } \\
\text { identity }\end{array}$ & $\begin{array}{l}\text { Oligo- } \\
\text { state }\end{array}$ & $\begin{array}{l}\text { Found } \\
\text { by }\end{array}$ & Method of resolution & $\begin{array}{l}\text { Seq } \\
\text { similarity }\end{array}$ & Range & Coverage & Description \\
\hline $1 \cup 02$ & 35 & $\begin{array}{l}\text { Mono } \\
\text { mer }\end{array}$ & Blast & $\begin{array}{l}\text { X-ray } \\
1.92 \mathrm{~A}^{\circ}\end{array}$ & 0.5 & $105-350$ & 0.7 & Trehalose-6-phosphate phosphatase-related protein \\
\hline
\end{tabular}


Table 4 Representative homology model in comparison to $1 \cup 02$

\begin{tabular}{lllll}
\hline Model & Built with & $\begin{array}{l}\text { Oligo } \\
\text { state }\end{array}$ & Ligands & QMEAN \\
\hline $\begin{array}{l}\text { Homology model } \\
\text { of TPPs from Swiss }\end{array}$ & Promod version 3.7 & Monomer & None & -3.27 \\
modeler & & & & \\
\hline
\end{tabular}

active site and coordinates the magnesium ion required for catalysis [37]. The results of MSA of 12 TPP sequences for the identification of conserved active site regions were as shown in Fig. 1a-c. The results were in agreement with conservation of regions in TPP sequences from plants, pathogenic bacteria, fungi, and nematodes. A similar study of conserved residues detection using CONSURF TOOL (comparison to Clustal Omega used in this study) was reported in more general haloacid dehalogenase family of proteins. The study reveals the conservation of aspartate and lysine residues similar to TPPs used in the present study [38].

\subsubsection{Phylogenetic tree construction}

The phylogenetic tree (phylogram) in Fig. 2 obtained from multiple sequence alignment data of Clustal W2 tool shows the evolutionary relationship between the various organisms as it was a rooted tree. Though the organisms were distinct as whole length complete sequence identity was not there, but are related mainly because of the conservation of the TPP sequences at the active site regions. As far as the evolution of TPP proteins is concerned, sequences are more conserved between plants and pathogenic bacteria which in turn have a close relationship with fungal TPPs followed by nematode TPPs. It seems probable that bacteria developed these genes at the beginning of evolution to face challenges like stress tolerance and pathogenecity. Later divergently plants and fungi started producing this. In plants, the role of these genes primarily is a developmental one, but under stress conditions, the same genes might be used for hyperproduction of trehalose. Fungi somehow mainly use trehalose pathway genes, especially TPPs for pathogenecity in different ways. Few nematodes had picked up these genes later through the link with fungi empowering themselves for development and pathogenecity. One more piece of information, it has been found that most of TPPs mentioned above are specific about natural substrate trehalose phosphate [38].

\subsubsection{Homology model development}

Homology model construction of representative TPP in comparison with 1U02 TPP was done with Swiss modeler server [30]. Overall, 664 templates were found. The model with the specific characters was built as in comparison to 1U02-A TPP from Thermophilus acidophilum shown in Tables 3 and 4. Figure 3a represents 1U02 3D structure retrieved from PDB, in comparison to which a homology model was built and shown in Fig. 3 b.

The representative homology model was incompatible with the 3D form of trehalose-6-phosphate phosphataserelated protein from $T$. acidophilum with a Qmean scoring function of -3.27 . In another study on haloacid dehalogenase protein modeling, a similar modeler called ITASSER was used to construct a representative model for binding studies. The structure prediction results were almost similar to Swiss modeler server used in the present study [38].

\subsection{Docking studies using AutoDock Vina 4.2 3.3.1 Preparation of modeled TPP and 17 ligands for docking}

AutoDock Vina 4.2 software contains tools for optimization of protein as well ligands such as assigning atomic charges
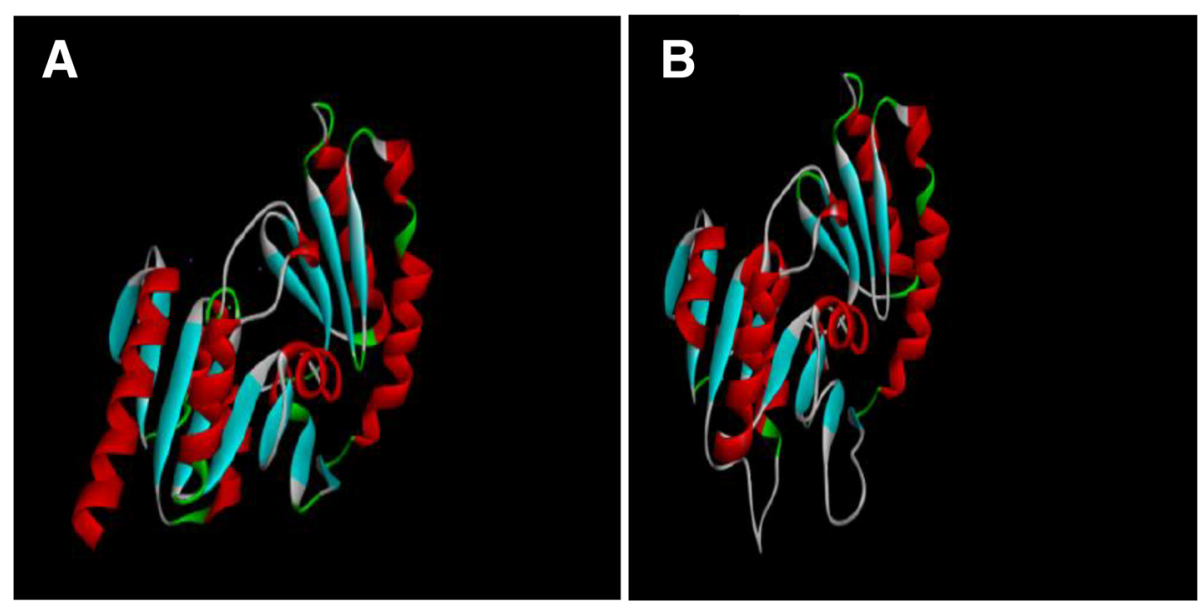

Fig. 3 a 3D structure of TPP (1U02)-related protein from PDB. b Representative Homology model of pathogenic TPP from Swiss modeler 


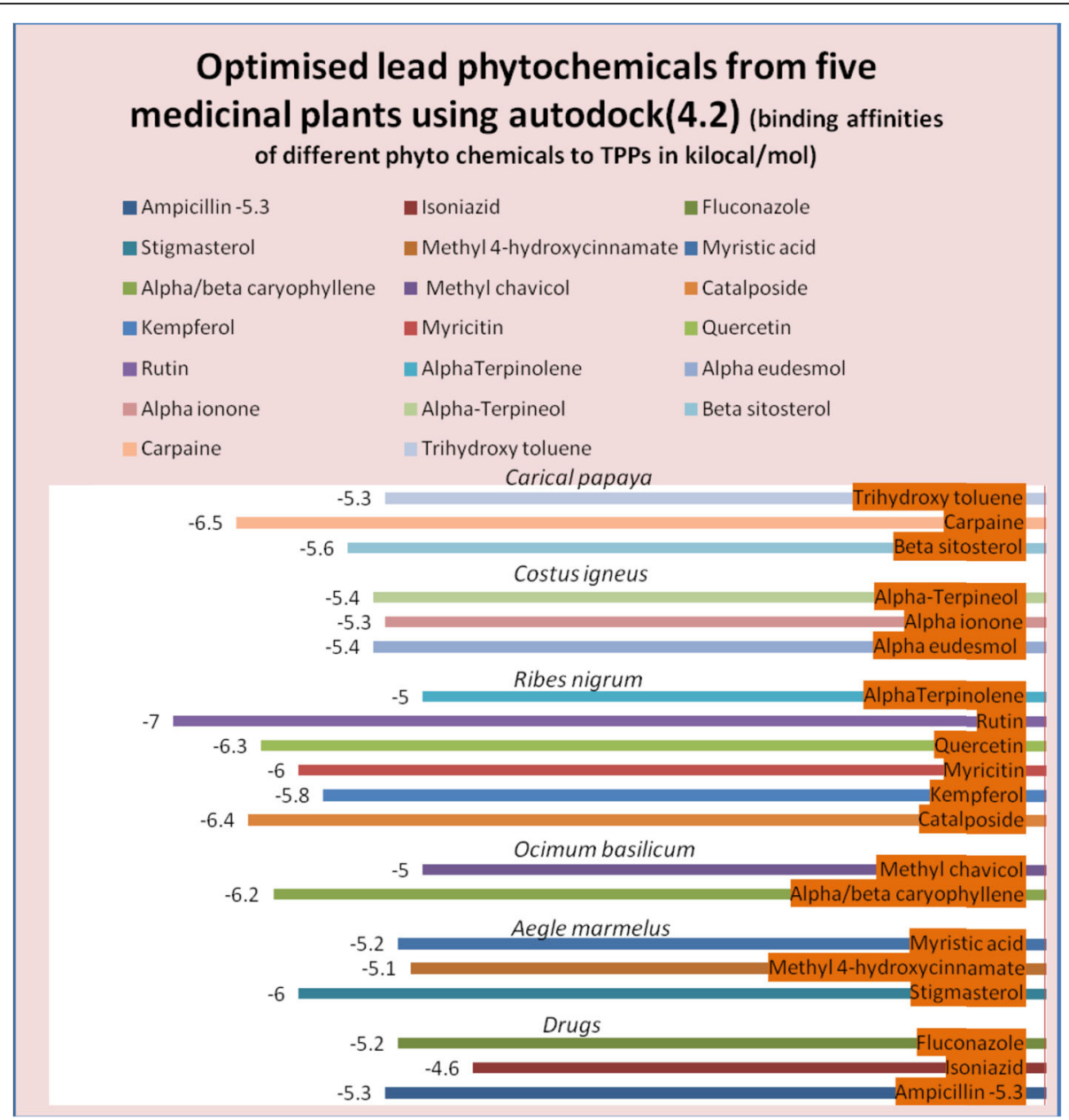

Fig. 4 Results of docking using AutoDock Vina 4.2

to make protein more polar, ligand modifications through charge and rotatable bonds assignment, calculation of energy contribution of desolvation during ligand-binding on protein, prior assigning of grid maps on protein surface for interaction with ligands by auto grid. The above facilities improve the speed, accuracy, docking with a new scoring

Table 5 Predicted best interaction for Modeled TPP using Autodock 4.2

\begin{tabular}{lll}
\hline Plant & $\begin{array}{l}\text { Inhibitor/drugs with best } \\
\text { interaction }\end{array}$ & $\begin{array}{l}\text { Binding energy- } \mathbf{~} \mathrm{G} \\
\text { (kcal/mole) }\end{array}$ \\
\hline Ribes nigrum & Rutin & -7.0 \\
Carica papaya & Carpaine & -6.5 \\
Ocimum basilicum & Caryophyllene & -6.2 \\
Aegle mermelos & Stigmasterol & -6.0 \\
Costus igneus & a-eudesmol & -5.4 \\
Drugs & Ampicillin (antibacterial) & -5.3 \\
& Fluconazole (antifungal) & -5.2 \\
& Isoniazid (antimycobacterial) & -4.6 \\
\hline
\end{tabular}

function, efficient optimization, and multithreading of molecular docking [33].

\subsubsection{Docking protein (modeled TPP) with ligand (phytochemicals) molecules}

In our present work, we have determined the docking or binding-free energy as shown in Fig. 4 which reflects the binding affinity of 17 ligands and 3 prescribed drugs to model TPP. The above docking studies signify the fact that out of seventeen phytochemicals Rutin shows the highest binding affinity of $-7.0 \mathrm{kcal} /$ mole and methyl chavicol with least among 17 phytochemicals was -5 . The prescribed drugs were reported to have binding energy equal to most of the phytochemical ligands tried. Therefore we have selected at least one phytochemical ligand which shows better docking energy from each of the plant. The ligands with best possible affinity to model TPP were represented in Table 5 to carry further studies [39]. Glide is the drug discovery tool of Schrodinger program used in a similar study alternate to AutoDock Vina of the present work. AutoDock Vina 


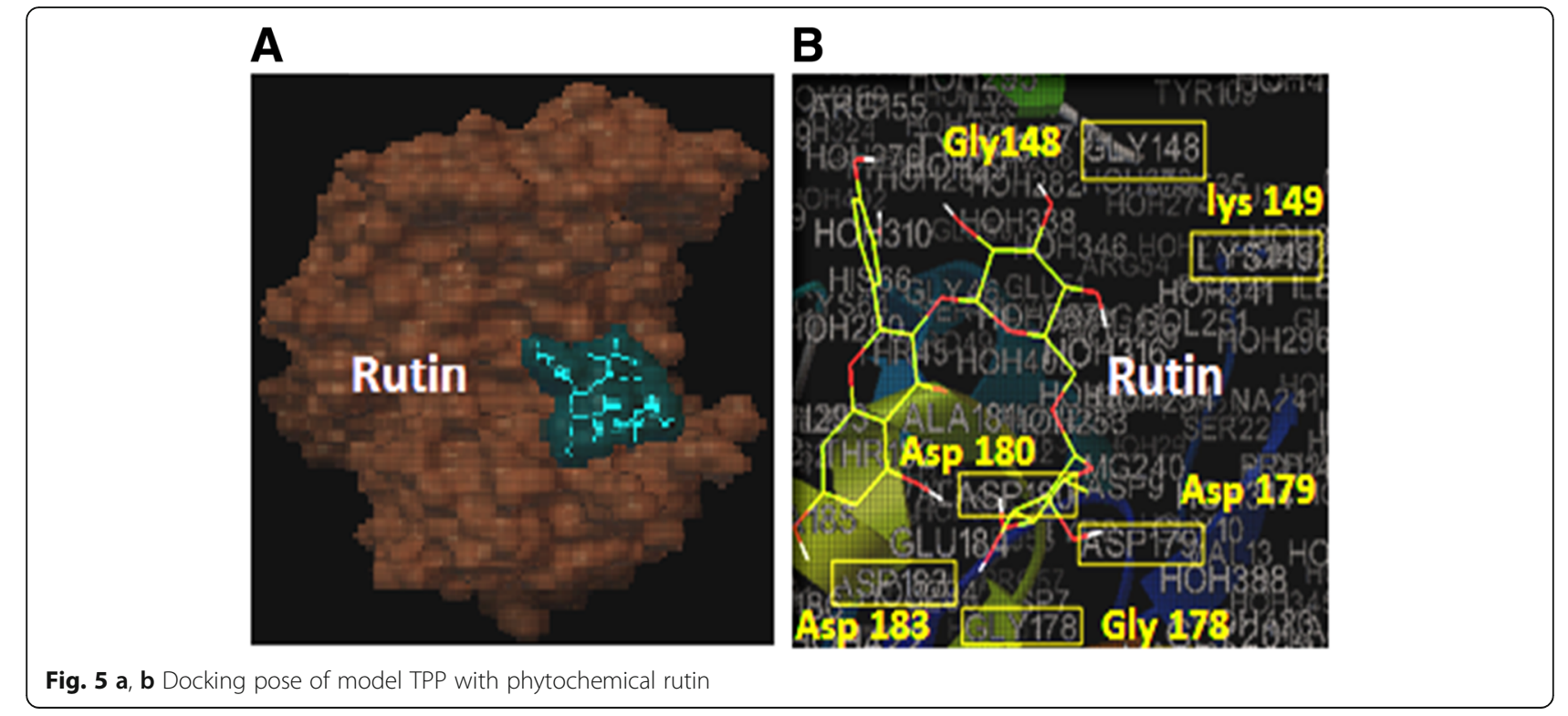

B

used in the study of docking calcinurin with inhibitors predicts binding affinity energy between -4.9 and -8.4 $\mathrm{kcal} /$ mole almost identical to the results of the present study [40].

\subsubsection{Model TPP docking poses with lead phytochemicals,} prescribed drugs, and substrate trehalose-6-phosphate

Blast P and MSA of various TPP sequences have helped us to know and confirm the conservation of active site regions. It has been said that HAD family of halogenases-like TPPs have a common reaction mechanism due to conserved active site regions. Further, we wanted to know the proximity of binding of each of the ligand on conserved regions of model TPP. The binding interactions of TPP with small molecules are shown in
Figs. 5, 6, 7, 8, 9, 10, 11, 12, 13. To our surprise, the poses of all ligands with TPP were in close proximity with region 3 of conserved active and binding sites [37]. The region consisting of Gly 148, Lys 149 further to 1645 amino acid away Gly 178, Asp 179, Asp 180, and Asp 183. This sequence seems to be the part of the active site and coordinates the magnesium ion required for catalysis. Drug molecule ampicillin was found to be in proximity with Lys 149, Asp 179, and Asp 180 compared to ligands. Fluconazole was close to Gly 148 and Lys 149 whereas antimycobacterial drug isoniazid bound to protein in between active site amino acids. Natural substrate trehalose-6-phosphate of all TPPs coordinates with most of the active site amino acids except Gly 148. In the case of phytochemicals, most of the active site amino
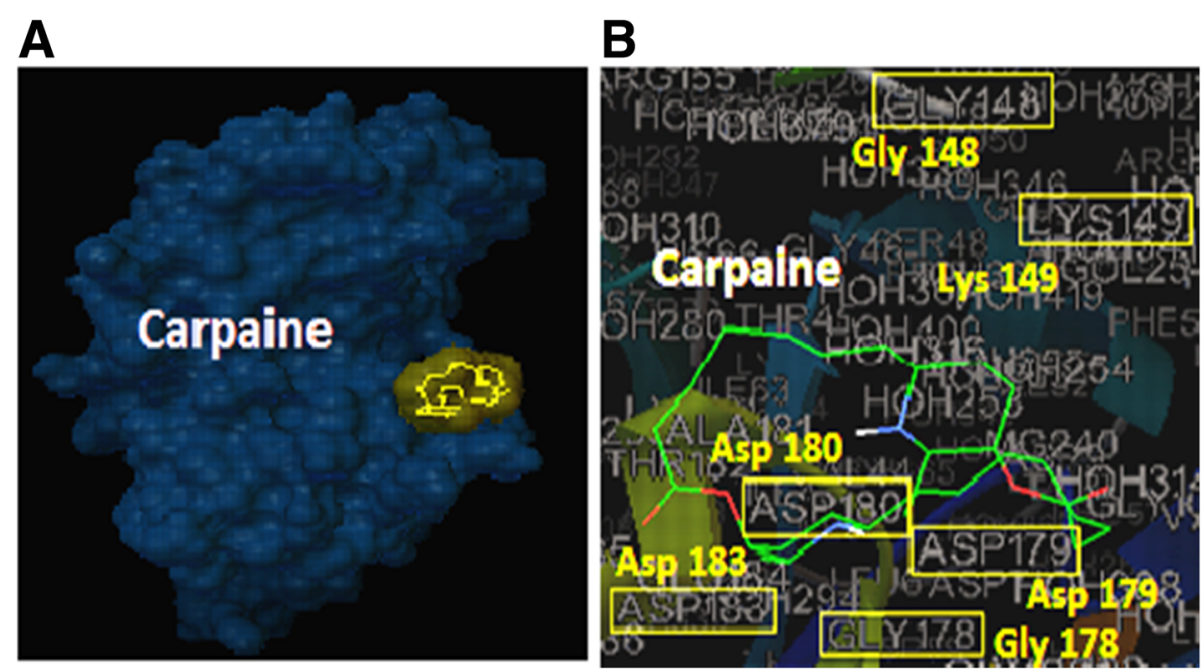

Fig. $\mathbf{6}$ a, b Docking pose of model TPP with phytochemical carpaine 

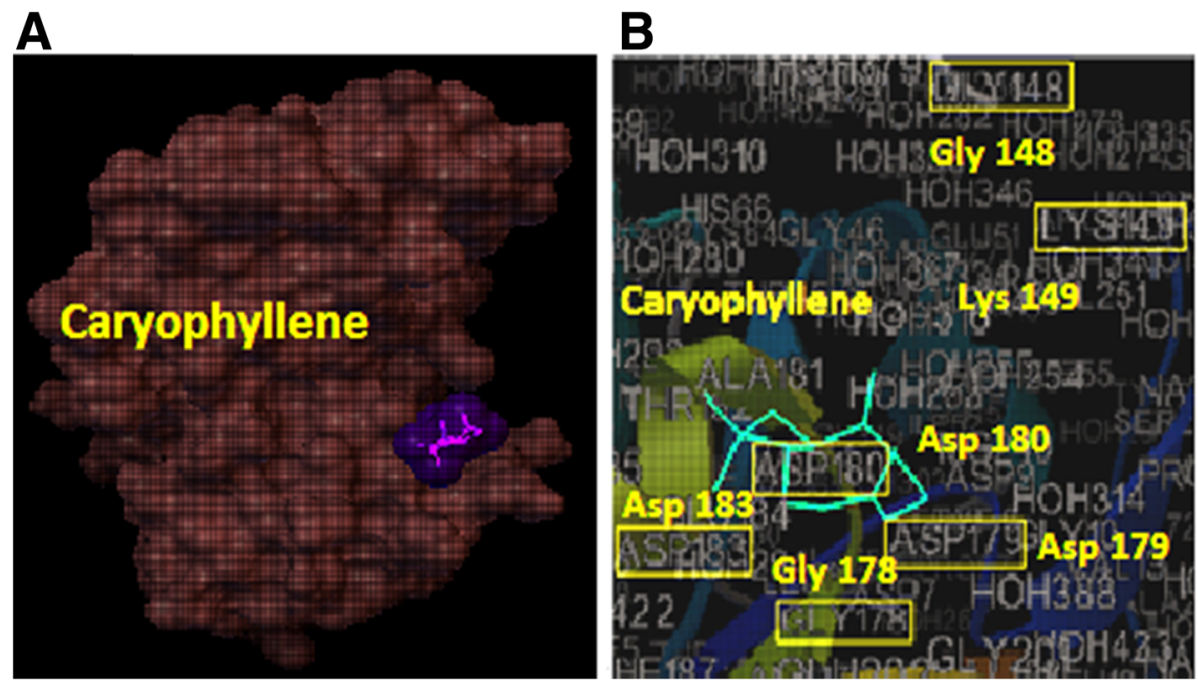

Fig. 7 a, b Docking pose of model TPP with phytochemical rutin caryophyllene

acids were within the distance range from 1.796 to 2.747 $\mathrm{A}^{\mathrm{o}}$. Glide is the drug discovery tool of Schrodinger program used in a similar study alternate to AutoDock Vina of the present work. Glide predicts $1-7 \mathrm{~A}^{\mathrm{o}}$ distance between the active site amino acids of calcineurin protein and inhibitory peptides. It seems to be less accurate but similar to the present study done using AutoDock Vina [41]. The conclusion that can be drawn by comparing binding affinity energies and binding poses, that the ligands bind active site cleft better than prescribed drugs.

\section{Discussion}

Trehalose phosphate phosphatases are known to play important roles in the development and virulence of many bacterial, fungal and nematode infections mentioned earlier. Trehalose pathway in general and TPPs in particular offer an excellent platform for drug designing as trehalose pathway is totally not present in animals [42]. Hence, TPP is a promising likely target molecule not only in the management of pathogenic microbes but also it may nullify the occurrence of multiple resistant strains.

Extensive literature survey was done to identify and select the phytochemicals against TPPs based on their medicinal properties for drug designing using docking. The six compounds (rutin, quercetin, myricitin, kaempferol, catalposide, terpinolene) from Ribes nigrum selected based on (high polyphones) antioxidant, antifungal (Candida albicans), and antibacterial potential. From Carica papaya, three compounds (tri hydroxy toluene, carpaine, sitosterol) were finalized as they are known to involve in antifungal (Aspergillus flavus and C. albicans),
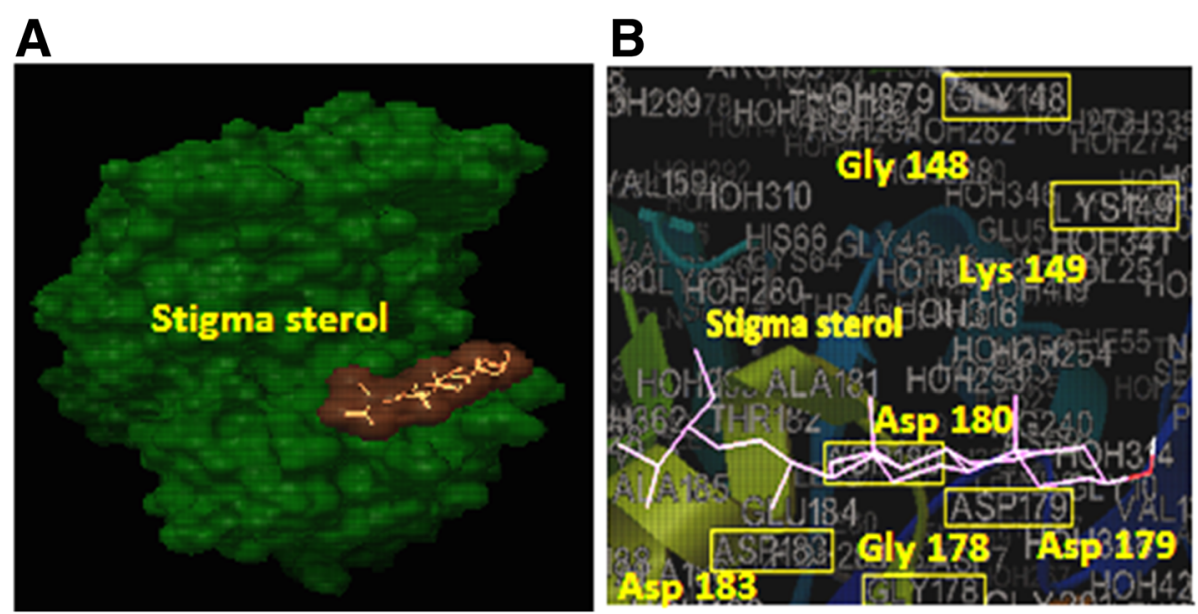

Fig. 8 a, b Docking pose of model TPP with phytochemical stigmasterol 


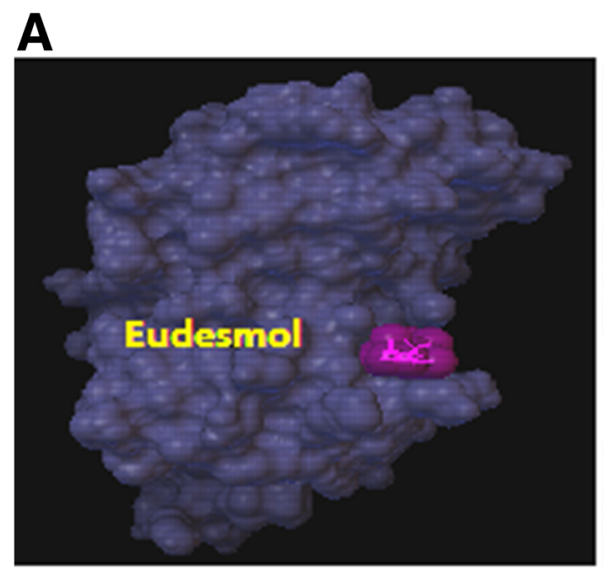

B

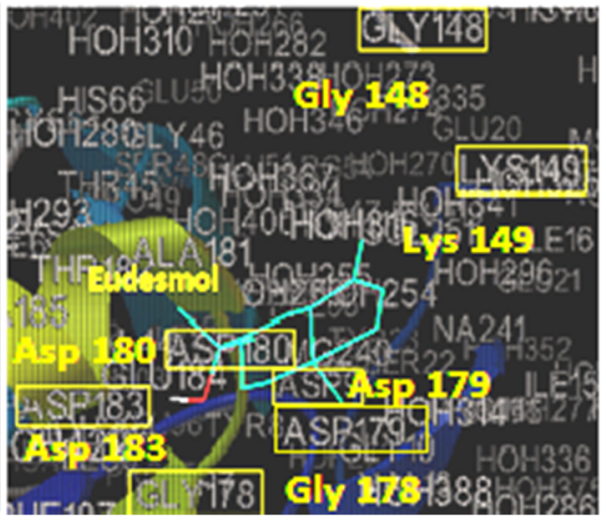

Fig. 9 a, b Docking pose of model TPP with phytochemical eudesmol

antituberculosis, nematicidal, and antidengue activities. Aegle mermelus phytochemicals (myristic acid, methyl 4 hydroxycinnamic acid, and stigmasterol) were known to exert antibacterial, antienzymatic, antifungal activities. Ocimum basilicum-derived compounds (methyl chavicol and caryophyllene) were reported as antifungal, antibacterial, cytotoxic, antimycobacterial, and anti-bovis. Lastly, three compounds ( $\alpha$ terpinol, ionone, eudesmol) from Costus pictus were antidiabitic, hypolipidimic, diuretic, antimicrobial, anticandidal, and anticancerous [1921, 23, 24]. Phytochemicals with such high medicinal values can be anticipated to possesS anti-TPP activity also as the TPP group of enzymes are involved in infection and pathogenecity in many bacteria, fungi, and nematodes.

TPPs belong to the HAD superfamily of magnesiumdependent phosphatases, which are distributed in both prokaryotes and eukaryotes. This superfamily uses a common catalytic reaction mechanism characterized by having three highly conserved motifs [43, 44]. MSA and phylogenetic tree analysis work confirms that phosphatase activity motifs of these halo acid halogenase family of TPPs are conserved in normal and pathogenic organisms and TPP-related genes are absent in animals. This forces us to propose that an inhibitor designed for TPP of one organism can be extrapolated to TPPs of other organisms and have no effect on animal systems. Our present studies were the investigations in this direction by in silico methods [38].

The representative homology model of TPP from Swiss modeler seems to be incompatible and consistent with the 3D structure of trehalose-6-phosphate phosphatase-related protein from $T$. acidophilum in many aspects except that it is devoid of ligand $\mathrm{Mg}^{2+}$ which is indicated by Qmean scoring function (-3.27). Model formation was done after following extensive methodologies like template search, template selection, model building, model quality estimation, ligand
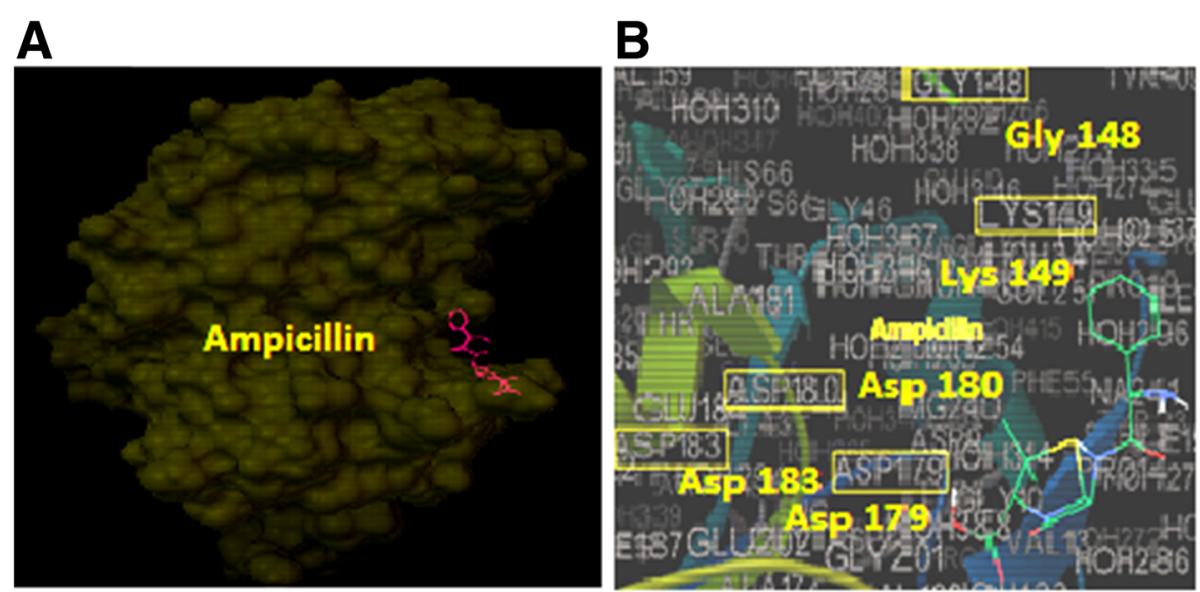

Fig. 10 a, b Docking pose of model TPP with ampicillin 

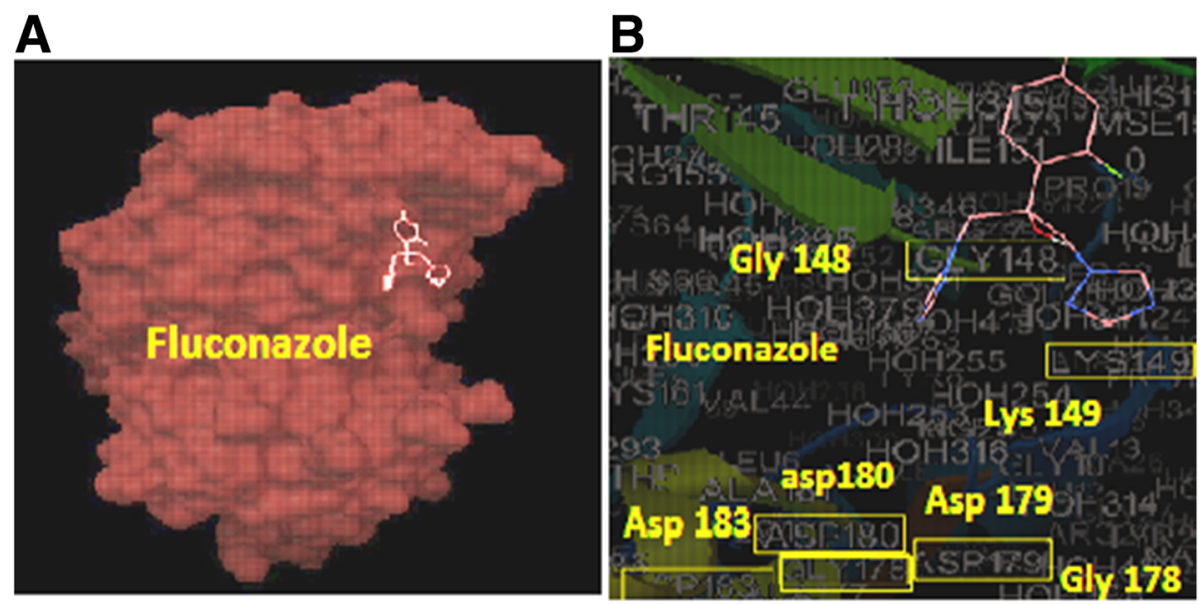

Fig. 11 a, b Docking pose of model TPP with antifungal fluconazole

modeling, and oligomeric state conservation. The minimum changes regarding the overall secondary and tertiary structures were reported $[30,38,45]$.

The concept of free energy $(\delta G)$ is used to determine the binding affinity of protein-ligand complex in docking studies using drug discovery tool AutoDock Vina 4.2. The negative and low value of $\delta G$ indicates the strong binding affinity between protein-ligand and that the ligand is in the most favorable conformation [41]. In this regard, poses of TPP binding by 5 ligands, at least 1 (with highest binding energy) from each of 5 plants, 3 drugs, and substrate trehalose phosphate were analyzed. In reality, we have not set any specific amino acid as rigid precision though the availability of some information on active site amino acid residues. Rather docking precision was set to regular precision and flexible ligand docking mode was employed. All the interaction studies showed the binding of leads to region 3 of the conserved active site indicating the importance of the region as it is specific for $\mathrm{Mg} 2+$ binding and significant part of the active site of TPPs [40].

Thus, TPP inhibition work offers scope for the development of potent next-generation drugs like rutin (in this study) which would help cure or effectively manage toxicities of pathogens. Phytochemicals from herbal sources seem to be real good agents in drug designing. Screening of a number of phytochemicals as potent inhibitors of TPPs was made easy with in silico studies using online and offline drug-designing programs such as Auto dock and Schrodinger. One more advantage of this in silico work is, it leads to screening more probable anti-TPP leads. In addition, phytochemical components may act like new generation drugs as well as cocktail of inhibitors along with the existing ones to manage diseases like tuberculosis, fungal infections, elephantiasis, and meningitis with minimum risk factors.
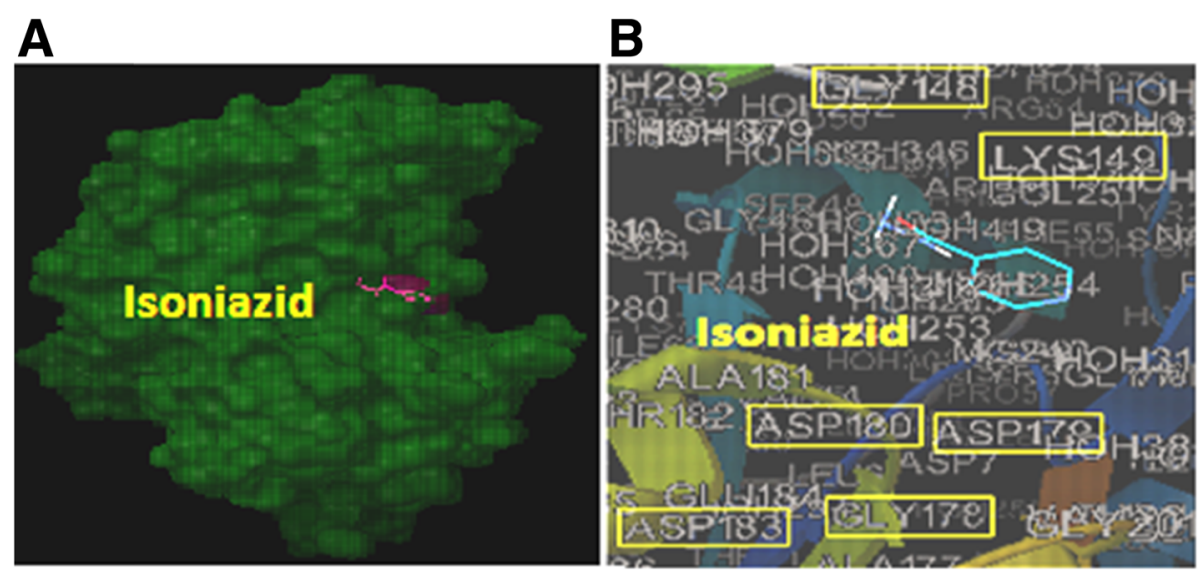

Fig. 12 a, b Docking pose of model TPP with isoniazid 


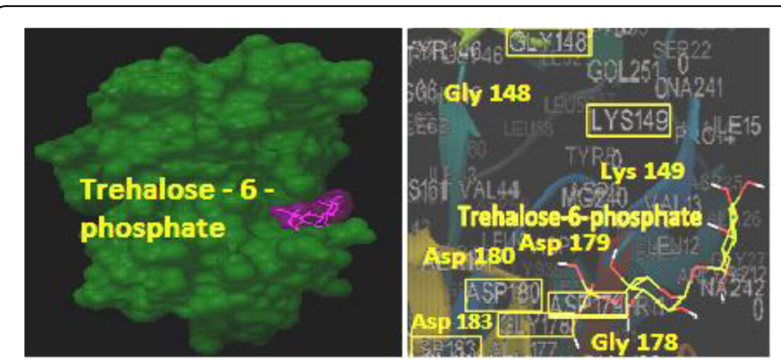

Fig. 13 a, $\mathbf{b}$ Docking pose of model TPP with substrate trehalose-6-phosphate

\section{Conclusion}

In the context of therapeutic and other potential applications, the enzymes of trehalose metabolism in particular that of TPP has become an attractive candidate to study functional mechanisms and to design drugs. Although the structures and catalytic mechanisms of several other phosphatases are well understood TPP has been largely ignored so far and there is still a lot of work to be done. There are specific advantages in choosing TPPs as a drug target. Since TPP binds only the small molecule ligand that makes it simpler to mimic the substrate by the inhibitor and avoids complications arising out of secondary recognition sites, like other phosphatases. Structural studies of few TPPs revealed that active site is present within a crevice that seems to bind substrate with high specificity and consequently high-affinity inhibitors can be designed. Our present in silico molecular modeling study using phytochemicals as inhibitors of TPPs justifies the above statement.

At present, we assume that the representative TPP targets chosen here for inhibitor design apply to other homology TPPs from pathogenic organisms. To support this hypothesis, we have done homology modeling and multiple sequence alignment study and show that active sites are conserved.

Our docking studies confirm a tight binding of phytochemicals to model protein active site and coordinate with essential Mg2+. Since all TPPs accept the same substrate, the mechanism and binding at the catalytic site are likely to remain the same for all the TPPs. In this context, our in silico studies need to be validated by in vitro work.

Finally, we have given the overall view of possible therapeutic relevance of selected pathogenic TPPs. In the future, it will be realized that the inhibition of TPP is a very valuable tool in research, and in silico-based in vitro work will reduce the effort of inhibitor design. Nevertheless, pharmaceutical researchers have considered TPPs as prime candidates for drug development, but still the field is in its infancy. Given the role of TPPs in innumerable biological processes, it appears almost certain that TPP inhibitors will be an integral part of the drugs of the next generation.

\section{Abbreviations}

1UO2: PDB ID of trehalose phosphate phosphatase related protein; Blast $P$ : Basic Local Alignment Search Tool; EBI: European Bioinformatics Institute.; EMBL: European Molecular Biology Laboratory; HAD: Halo acid dehalogenases family; LogP: Lipophilicity; M. smegmatis: Mycobacterium smegmatis; M. tuberculosis (MT): Mycobacterium tuberculosis; Mg

${ }^{2+}$ : Magnesium; Mol.Wt: Molecular weight; MSA: Multiple sequence alignment; $\mathrm{Nb}$ : No. of rotatabe bonds; $\mathrm{nOH}$ : No. of $\mathrm{H}$ bond acceptors; nOHNH: No. of H bond donors; PDB: Protein data bank; Qmean : Qualitative Model Energy ANalysis for protein; RA 3: Ramosa 3; T6P: Trehalose-6phosphate; TB: Tuberculosis; Torsdorf: Torsional degrees of freedom of ligand; TPP: Trehalose-6-phosphate phosphatase; TPS: Trehalose-6-phosphate synthase; Vol: Molecular volume

\section{Acknowledgements}

The authors express thanks to the staff members of Biochemistry Department, Dayananda Sagar College, Bangalore for their valuable advice and help.

\section{Authors' contributions}

$\mathrm{HR}$ carried out sequence alignment work, phylogenetic studies, procurement of $3 \mathrm{D}$ model of protein, and drug discovery work. KV participated in the design of work and helped to draft the manuscript. KS participated in the sequence alignment and phytochemical work and provided valuable inputs in writing the manuscript. All authors have read and approved the manuscript.

\section{Funding}

Worked with online and freely available softwares.

Availability of data and materials

The data and material are part of Ph.D. thesis. Hence, not deposited in repositories.

\section{Ethics approval and consent to participate}

Not applicable

\section{Consent for publication}

Not applicable

\section{Competing interests}

The authors declare that they have no competing interests.

\section{Author details}

${ }^{1}$ Research and Development Centre, Bharathiar University, Coimbatore 641046, India. ${ }^{2}$ Post graduate Department of Biochemistry, Dayananda Sagar College of Biosciences, Bangalore 560078, India. ${ }^{3}$ Department of

Biochemistry, Karnatak University, Dharwad, Karnataka 580003, India.

Received: 10 July 2019 Accepted: 4 December 2019

Published online: 03 February 2020

\section{References}

1. Crowe JH, Crowe LM, Chapman D (1984) Preservation of membranes in anhydrobiotic organisms: the role of trehalose. Science. 223:701-703

2. Kaasen I, McDougall J, Strom AR (1994) Analysis of the otsBA operon for osmoregulatory trehalose synthesis in Escherichia coli and homology of the OtsA and OtsB proteins to the yeast trehalose-6-phosphate synthase/ phosphatase complex. Gene 145:9-15

3. Elizabeth WP, Uwe H, Eleftherios M, Thomas R, Dena T (2006) Characterization and Regulation of the Trehalose Synthesis Pathway and Its Importance in the Pathogenicity of Cryptococcus neoformans. Infect Immun 74(10):5877-5887

4. Helen NM, Graham RS, Vladimir VM, Alexander SA, Richard H (2005) The OtsAB pathway is essential for trehalose biosynthesis in Mycobacterium tuberculosis. The Journal Of Biological Chemistry 280(15):14524-14529

5. Kormish JD, McGhee JD (2005) The C. elegans lethal gut-obstructed gob-1 gene is trehalose-6-phosphate phosphatase. Dev Biol. 287(1):35-47

6. Mykola MM, Larissa DR, Miguel R, Rosalia DO, Johan MT, Patrick VD (2008) Combined Inactivation of the Candida albicans GPR1 and TPS2 Genes 
Results in Avirulence in a Mouse Model for Systemic Infection. Infect Immun. 76(4):1686-1694

7. Nadia AB, Ghyslaine V, Hong L, Fabrice NG, Mirjam U (2010) Role of trehalose biosynthesis in Aspergillus fumigatus development, stress response, and virulence. Infect Immun. 78(7):3007-3018

8. Popchai N, Uwe H, Julia AB, Christabel W, Methee C (2009) The trehalose synthesis pathway is an integral part of the virulence composite for Cryptococcus gattii. Infect Immun. 77(10):4584-4596

9. Susheela K, Prashant KS, Mohd S, Manisha P, Shailja MB (2012) In vitro silencing of Brugia malayi trehalose-6-phosphate phosphatase impairs embryogenesis and in vivo development of infective larvae in jirds. PLoS Negl Trop Dis. 6(8):e1770

10. Tobias S, Kristine B, Fong FH, Gitta H, Otto H (2013) Diversion of phagosome trafficking by pathogenic Rhodococcus equi depends on mycolic acid chain length. Cell Microbiol. 15(3):458-473

11. Dye C, Scheele S, Dolin P, Pathania V, Raviglione MC (1999) Consensus statement. Global burden of tuberculosis: estimated incidence, prevalence, and mortality by country. WHO Global Surveillance and Monitoring Project. JAMA 282(7):677-686

12. Kuni T, Cindy W, Gurdyal SB (2005) Pathway to synthesis and processing of mycolic acids in Mycobacterium tuberculosis. Clinical Microbiology Reviews 18(1):81-101

13. Vivek R, Nagatoshi F, Steven AP, Michael SG (2005) Mycobacterium tuberculosis controls host innate immune activation through cyclopropane modification of a glycolipid effector molecule. Journal of experimental medicine 201(4):535-543

14. Martínez EM, Martínez VE, González PP, Ros JM, Garcca PP, Arguelles JC (2009) Role of trehalose-6P phosphatase (TPS2) in stress tolerance and resistance to macrophage killing in Candida albicans. Int J Med Microbiol. 299(6):453-464

15. Serneels J, Tournu H, Van DP (2012) Tight control of trehalose content is required for efficient heat-induced cell elongation in Candida albicans. J Biol Chem. 287(44):36873-36882

16. Zaragoza O, De VC, Ponton J, Gancedo C (2002) Disruption in Candida albicans of the TPS2 gene encoding trehalose-6-phosphate phosphatase affects cell integrity and decreases infectivity. Microbiology. 148(5):1281-1290

17. Avonce N, Mendoza-Vargas A, Morett E, Iturriaga G (2006) Insights on the evolution of trehalose biosynthesis. BMC Evol Biol. 6:109-124

18. Liu C, Dunaway-Mariano D, Mariano PS (2017) Rational design of reversible inhibitors for trehalose 6-phosphate phosphatases. Eur J Med Chem. 128: 274-286

19. Beena J, Joji R (2010) Analysis of the essential oils of the stems, leaves and rhizomes of the medicinal plant costus pictus from southern india. International journal of pharmacy and pharmaceutical sciences issn-0975$14912(2): 100-101$

20. Farina M, Preeti B, Neelam P (2014) 2014, Phytochemical evaluation, antimicrobial activity, and determination of bioactive components from leaves ofAegle marmelo. BioMed Research International Volume 497606:11

21. Hossain MA, Kabir MJ, Salehuddin SM, Rahman SM, Das AK, Singha SK, Alam MK, Rahman A (2010) Antibacterial properties of essential oils and methanol extracts of sweet basil Ocimum basilicum occurring in Bangladesh. Pharm Biol. 48(5):504-511

22. Kapil S, Petit C, Drago VN, Ronning DR, Sucheck SJ (2019) Synthesis and in Vitro Characterization of Trehalose-Based Inhibitors of Mycobacterial Trehalose 6-Phosphate Phosphatases. Chembiochem 20(2):260-269

23. Singh O, Ali M (2011) Phytochemical and antifungal profiles of the seeds of carica papaya L. Indian J Pharm Sci. 73(4):447-451

24. Tatjana S, Katarina S, Mihailo R, Gordana Z, Teodora J (2010) Composition and antimicrobial activity of the essential oil of the leaves of black currant (Ribes nigrum L.) cultivar Cacanska crna. J. Serb. Chem. Soc. 75(1):35-43

25. QikProp (2012) version 3.5, Schrödinger. LLC, New York

26. Lipinski CA (2004) Lead- and drug-like compounds: the rule-of-five revolution. Drug Discovery Today: Technologies. 1(4):337-341

27. Sievers F, Andreas W, David D, Toby JG, Kevin K, Weizhong L, Rodrigo L, Hamish M, Michael R, Johannes S, Julie DT, Desmond GH (2011) Fast, scalable generation of high-quality protein multiple sequence alignments using Clustal Omega. Molecular Systems Biology 7:539

28. Larkin MA, Blackshields G, Brown NP, Chenna R, McGettigan PA et al (2007) ClustalW and Clustal X version 2. Bioinformatics. 23(21):2947-2948
29. Łopieńska-Biernat E, Molcan T, Paukszto Ł, Jastrzębski JP, Myszczyński K (2018) Modelling studies determing the mode of action of anthelmintics inhibiting in vitro trehalose-6-phosphate phosphatase (TPP) of Anisakis simplex s.l. Exp Parasitol. 184:46-56

30. Rao KN, , Kumaran D, Seetharaman J, Bonanno JB, Burley SK, Swaminathan S. Crystal structure of trehalose-6-phosphate phosphatase-related protein: biochemical and biological implications. Protein Sci. 2006;15: 1735-1744

31. Namiko SN, Nobuhiro N, Simon M, Hajime S, Jackson D (2006) A trehalose metabolic enzyme controls inflorescence architecture in maize. Nature 441: 227-230

32. Marco B, Stefan B, Andrew W, Konstantin A, Gabriel S, Tobias S, Florian K, Tiziano GC, Martino B, Lorenza B (2014) Torsten S SWISS-MODEL: modeling protein tertiary and quaternary structure using evolutionary information. Nucleic Acids Res. 42(W1):W252-W258

33. Oleg T, Arthur JO (2010) Auto Dock Vina: improving the speed and accuracy of docking with a new scoring function, efficient optimization, and multithreading. Journal of Computational Chemistry 2010 31(2):455-461

34. Huey R, Morris GM, Olson AJ, Good Sell DS (2007) A semi empirical free energy force field with charge based desolvation. J of compt Chem 28: 1145-1152

35. Garrett MM, David SG, Robert SH, Ruth H, William EH (1998) Automated docking using a lamarkian genetic algorithm and empirical binding free energy function. J compt Chem 19:1639-1662

36. Chaitra P, Jeyanthi GP (2011) Insilico drug designing approaches for latent autoimmune diabetes in Adults (LADA). International Journal of Pharma and Bio Sciences 2:B16-B27

37. Dong HS, Anne R, Jaru J, Hisao Y, Rosalind K, David EW, Sung HK (2003) Crystal structure of a phosphatase with a unique substrate binding domain from Thermotoga maritima. Protein Science 12:1464-1472

38. Raghunath S, Konkimalla VB, Jagnyeswar R (2015) In silico phylogenetic analysis and molecular modelling study of 2-haloalkanoic acid dehalogenase enzymes from bacterial and fungal origin. Advances in Bioinformatics. 2016:1-10

39. Tyagi R, Verma S, Mishra S, Srivastava M, Alam S, Khan F, Srivastava SK (2019) In Vitro and In Silico Studies of Glycyrrhetinic Acid Derivatives as AntiFilarial Agents. Curr Top Med Chem. 19(14):1191-1200

40. Harish BM, Devaraju KS, Gopi A, Saraswathi R, Babu RL, Chidananda Sharma S (2013) In silico binding affinity study of calcineurin inhibitors to calcineurin and its close associates. Indian Journal of Biotechnology. 12:213-217

41. Devaraju KS, Harish BM, Saraswathi R (2014) Molecular Docking Study of Auto Inhibitory Domain Fragments to Calcineurin A. International Journal of Scientific \& Engineering Research 5(1):2095-2100

42. Yu Y, Zhang H, Zhu G (2010) Plant-type trehalose synthetic pathway in cryptosporidium and some other apicomplexans. PLoS ONE 5(9):e12593. https://doi.org/10.1371/journal.pone.0012593

43. Morais MC, Zhang W, Baker AS, Zhang G, Dunaway-Mariano D, Allen KN (2000) The crystal structure of Bacillus cereus phosphonoacetaldehyde hydrolase: Insight into catalysis of phosphorus bond cleavage and catalytic diversification within the HAD enzyme superfamily. Biochem 39:10385-10396

44. Suzuki Y, Gojobori T (1999) A method for detecting positive selection at single amino acid sites. Mol Biol Evol 16:1315-1328

45. Cross M, Lepage R, Rajan S, Biberacher S, Young ND, Kim BN, Coster MJ, Gasser RB, Kim JS, Hofmann A (2017) Probing function and structure of trehalose-6-phosphate phosphatases from pathogenic organisms suggests distinct molecular groupings. FASEB J. 31(3):920-926

46. Umesh HR, Ramesh KV (2016) Purification, characterization and partial structure determination of native trehalose-6-phosphate phosphatase from maize genotype EC 558706 under salt stress condition. Int j pharma and Bio Sci 7(3):(B)1263-1273

\section{Publisher's Note}

Springer Nature remains neutral with regard to jurisdictional claims in published maps and institutional affiliations. 